\section{$\underset{\substack{\text { hommes } \\ \text { \& migrations }}}{ }$}

\section{Hommes \& migrations}

Revue française de référence sur les dynamiques

migratoires

1277 | 2009

France - Allemagne

\title{
Naturalisations en Allemagne : progrès et retards
}

\section{Dietrich Thränhardt}

Traducteur : Fritz Wittek-Kaïm

\section{CpenEdition}

Journals

Édition électronique

URL : http://journals.openedition.org/hommesmigrations/164

DOI : 10.4000/hommesmigrations. 164

ISSN : 2262-3353

Éditeur

Musée national de l'histoire de l'immigration

Édition imprimée

Date de publication : 1 janvier 2009

Pagination : 68-78

ISSN : 1142-852X

Référence électronique

Dietrich Thränhardt, « Naturalisations en Allemagne : progrès et retards », Hommes \& migrations [En ligne], 1277 | 2009, mis en ligne le 29 mai 2013, consulté le 01 mai 2019. URL : http://

journals.openedition.org/hommesmigrations/164; DOI : 10.4000/hommesmigrations.164 


\section{Naturalisations en Allemagne: progrès et retards ${ }^{(1)}$}

Par Dietrich Thränhardt, professeur de sciences politiques, université de Münster

De 1982 à 1998, le nombre d'étrangers en l'Allemagne est passé de 4,7 à 7,3 millions, pendant qu'un difficile débat se déroulait pour aboutir à des réformes facilitant la naturalisation. Après les processus

d'intégration réussis par les secteurs économiques et sociaux, l'acquisition de la nationalité a été reconnue comme un nouveau moyen d'intégration. Néanmoins, même si les naturalisations ont augmenté, les taux restent faibles et en deçà des objectifs.

De nombreux obstacles demeurent comme l'interdiction des nationalités multiples selon les pays d'origine, l'application inégale de la loi selon les territoires ou la longueur des procédures. 


\section{Des déficits malgré un consensus de fond}

"La doctrine jadis dominante, et professée parfois encore aujourd'hui selon laquelle l'octroi de la nationalité participerait à la délimitation du Staatsvolk ${ }^{(2)}$ d'un point de vue d'ordre public et relèverait à ce titre de l'arbitraire décisionnel de l'État, n'est pas conforme aux principes de l'État de droit démocratique et social tel que le conçoit la Loi fondamentale."(3) Tels sont les termes utilisés dans un arrêt que la Cour constitutionnelle fédérale allemande pris en 1974, au sujet de la transmission de la nationalité non seulement par le père, mais aussi par la mère. Conformément à cette conception des droits civiques, la naturalisation est, en 1993, devenue un droit opposable en Allemagne, pour les personnes remplissant les conditions légales. Des principes élémentaires de démocratie exigent que les personnes étant durablement établies en Allemagne puissent acquérir la nationalité allemande et tous les droits et obligations politiques qui s'y attachent. Dans le cas contraire, il y aurait un déficit démocratique. Or, la qualité du processus politique est affectée si des catégories identifiables et importantes de la population ne bénéficient pas de droits politiques, ne peuvent accéder au statut de sujets politiques et restent des objets de la politique. Bien que des représentants de tous les partis politiques aient maintes fois fait remarquer que dans toute la mesure du possible le Staatsvolk et la population devraient se confondre, l'Allemagne continue à accuser un important déficit de naturalisations.

L'acquisition de la nationalité et la possession de droits civiques entiers sont des impératifs cruciaux de l'intégration. À l'époque du gouvernement Kohl, la question avait déjà fait l'objet d'un vif débat, car malgré la doctrine officielle qui proclamait que “l'Allemagne n'est pas un pays d'immigration", de 1982 à 1998, le nombre d'étrangers passait de 4,7 millions à 7,3 millions, amenant le gouvernement fédéral de l'époque et son ministre des Affaires Intérieures, Wolfgang Schäuble, à inviter les étrangers durablement établis sur le territoire de la République fédérale à se faire naturaliser.(4)

À l'époque où elle recrutait des travailleurs étrangers, et pendant les décennies suivantes, l'Allemagne avait fait des efforts considérables afin de promouvoir l'égalité sociale et économique des "travailleurs étrangers et de leurs familles"(terme officiel à l'époque), non sans obtenir des résultats qui, comparés à ceux d'autres pays européens, paraissent plutôt positifs. ${ }^{(5)}$ Particulièrement dans les dispositifs de la cogestion des entreprises, on a pu constater des niveaux élevés d'intégration et de participation, plus élevés même qu'on aurait pu initialement les anticiper. Dans ce domaine, l'intégration peut être considérée comme entièrement réussie, d'autant mieux qu'on rencontre un nombre surprenant de cadres syndicaux issus 
de l'immigration, comme le montre le tableau 1 ci-dessous. Ces résultats sont dus à une décision unanime du Bundestag de 1972, octroyant les droits de vote actifs et passifs entiers aux élections des représentants du personnel à tous les salariés, indépendamment de leur nationalité. Cette décision allait au-delà des normes européennes en vigueur, qui n'instauraient ces droits que pour les seuls ressortissants des États membres de ce qui était encore la CEE.

Tableau 1 : présidents et suppléants de Betriebsräte ${ }^{(6)}$ d'origine étrangère dans les entreprises relevant du syndicat IG Metall(7) de 1975 à 2006.

Source : IG Metall. Chiffres de 2006 pour une partie de l'année seulement. Graphique de l'auteur.

En revanche, l'idée de Heinz Kühn, premier délégué aux Affaires des Étrangers, qui proposa en 1979 d'octroyer la nationalité allemande à la deuxième génération, née et élevée en Allemagne, n'a pas rencontré de succès ; elle coula dans les vagues xénophobes qui marquèrent le climat des années 1980. Contrairement à ce qu'on a souvent pu lire dans la littérature de la fin du $\mathrm{XX}^{\mathrm{e}}$ siècle ${ }^{(8)}$, les faibles taux de naturalisation n'avaient pas grand-chose à voir avec des traditions ethniques allemandes ; elles résultaient de décisions et d'intérêts politiques qui résistaient efficacement aux tentatives de transformation. ${ }^{(9)}$

La première loi destinée à faciliter les naturalisations proposée par le ministre des Affaires Intérieures, Wolfgang Schäuble, fut adoptée dans la foulée du processus de réunification et entra en vigueur le $1^{\text {er }}$ janvier 1991. Initialement, elle ne concernait que les étrangers et leurs familles qui avaient jadis été recrutés par l'industrie allemande; de plus, sa durée de validité était limitée.

Dans le cadre de l'Asylkompromiss ${ }^{(10)}$, le SPD obtint en 1993 l'instauration d'un droit à la naturalisation et la suppression de la limitation de la durée de validité de la loi. Dans un premier temps, des réformes plus ambitieuses échouèrent ; la Kinderstaatszugehörigkeit ${ }^{(11)}$, annoncée en 1994 par le chancelier Helmut Kohl, 
contradictoire et controversée, ne fut pas instaurée. La réforme fondamentale du droit de la nationalité, prévoyant notamment l'octroi d'un droit à la nationalité allemande aux enfants nés en Allemagne et dont au moins un parent y vit légalement depuis huit ans, ne fut réalisée qu'après l'alternance de 1998.

Dès les années 1980, le SPD, le FDP et Les Verts avaient pris position pour une refonte de la naturalisation, et notamment pour un droit à la naturalisation dès la naissance en Allemagne. Il y avait déjà eu un débat comparable en 1913, qui s'était soldé par une défaite des sociaux-démocrates et des libéraux face aux conservateurs. ${ }^{(12)}$ Le premier grand projet du nouveau gouvernement rouge-vert fut une réforme fondamentale du droit de la nationalité qui allait comporter une ouverture très large, allant jusqu'à autoriser des nationalités multiples. La CDU s'y opposa en lançant un appel à signer une pétition, son argument principal étant que chacun doit opter pour une seule nationalité, et que le fait de pouvoir en posséder deux mettrait les étrangers dans une position privilégiée vis-à-vis des Allemands. Grâce à cette campagne, la CDU gagna les élections pour la diète de la Hesse, et le nouveau gouvernement perdit sa majorité à la deuxième chambre, le Bundesrat.

Pour sortir de l'impasse, le gouvernement fédéral rouge-vert passa un compromis avec les libéraux du FDP, compromis qui prévoit un droit à la nationalité allemande dès la naissance pour tous les enfants nés en Allemagne dont au moins un parent est légalement établi en Allemagne depuis huit ans ou plus. Cette règle va plus loin qu'en France, parce que le jus soli n'y vaut que pour les enfants dont les parents sont eux aussi nés en France. Cependant, il existe une importante limitation : entre 18 et 23 ans, les personnes concernées devront choisir entre la nationalité allemande et celle de leurs parents. À moins d'être abolie à temps, cette clause occasionnera d'importants coûts administratifs à partir de 2017, moment où elle deviendra opératoire.

Parallèlement, l'acquisition de la nationalité allemande a été facilitée ; aujourd'hui, elle est un droit opposable - au terme de huit années de séjour légal. Cependant, dans ces cas aussi, les requérants devront renoncer à leur ancienne nationalité. Beaucoup d'immigrés - notamment ceux de la première génération s'y refusent, que ce soit pour des raisons utilitaires, pour ne pas créer d'obstacle à un éventuel retour, ou pour des raisons identitaires, par attachement à leur pays d'origine. De plus, dans l'ordre juridique allemand, des étrangers légalement établis bénéficient très largement des mêmes droits que les nationaux : ils n'ont pas de raison de craindre une expulsion ou d'autres préjudices s'ils ne se font pas naturaliser à la différence par exemple des États-Unis.

Malgré le rôle central de la nationalité tant dans sa dimension utilitaire, comme condition de participation aux décisions politiques, que dans sa dimension 
identitaire, comme sentiment d'appartenance à la nation -, et malgré les grands efforts politiques qui ont été nécessaires pour faire aboutir ces réformes, il reste en Allemagne d'importants déficits en matière de naturalisation. Depuis dix ans, le nombre de naturalisation des résidents dotés d'une nationalité étrangère a cessé de croître (voir tableau 2 ci-dessous). Si les effets statistiques des entrées sur le territoire et des naissances d'enfants de nationalité étrangère d'un côté et des naturalisations de l'autre s'équilibrent à peu près, c'est surtout grâce à l'octroi automatique de la nationalité allemande aux enfants de parents étrangers, dont au moins un a légalement résidé pendant huit ans ou plus en Allemagne.

\section{Tableau 2 : naturalisations en Allemagne (1996-2006).}

Source : Office statistique fédéral 2007. Graphique de l'auteur.

\section{L'interdiction des nationalités multiples et quelques comparaisons européennes}

L'espoir de changements conséquents qui a motivé la réforme du droit de la naturalisation de 1999 et qui avait déjà été formulé lors de la réforme, beaucoup moins remarquée, de 1990-1993 ne s'est pas réalisé. Bien qu'en termes absolus le nombre de naturalisations soit plus grand qu'avant la réforme, une comparaison internationale montre que les taux de naturalisations, calculés en pourcentage des naturalisés au cours d'une même année sur l'ensemble des étrangers, restent extrêmement bas (voir tableau 3 ci-dessous). Beaucoup de pays réussissent mieux 
dans ce domaine, et parmi ceux-ci tous ne sont pas comme les États-Unis, le Canada ou l'Australie, des pays d'immigration traditionnels, mais aussi des pays parfaitement comparables à l'Allemagne comme la France, le Royaume Uni, la Suisse, les Pays-Bas et surtout la Suède, mais aussi des pays d'immigration plus récente comme l'Espagne, voire très récente, comme la Slovaquie.

Par conséquent, le nombre des étrangers en Allemagne (environ 6,7 millions) n'a quasiment pas changé depuis des années et reste à peu près deux fois supérieur à celui des étrangers en France, qui accueille un nombre comparable d'immigrés, mais qui pratique depuis des décennies une politique active de naturalisation. À partir de 2001, on constate même que, malgré la réforme controversée du droit de la naturalisation, le nombre des naturalisations recule, et il faut s'attendre à ce que l'introduction du test de naturalisation entraine un nouveau déclin.

\section{Tableau 3 : taux de naturalisations dans quelques} États européens 1996-2005.

Source : OECD, International Migration Outlook. SOPEMI 2007, Paris, 2007, p. 359. Graphique de l'auteur.

La raison de ce déclin est la suppression de la possibilité de préserver leur ancienne nationalité, pour les immigrés venant de Turquie, de l'ancienne Yougoslavie et de plusieurs autres pays. Après la première réforme de 1990-1993, les naturalisations de Turcs étaient passées de quelques milliers par an à 100324 en 1999. Un ressortissant turc sur vingt s'est fait naturaliser au cours de cette seule année. Depuis, le nombre a régulièrement reculé, se stabilisant seulement en 2006, autour de 33 000. Dans la même période, le taux de naturalisation des ressortissants turcs a chuté de $4,9 \%$ à $1,9 \%$. Il faut conclure de ces chiffres que, pour le plus important 
groupe d'immigrés en Allemagne, la réforme du droit de la nationalité en 1999 est restée sans effet. L'opinion publique allemande perçoit à peine les succès des politiques de naturalisation d'autres États, et encore moins les sentiments plus forts d'appartenance au pays d'immigration qui, comme le montrent les exemples de la Suède, du Canada ou des États-Unis, tendent à accompagner des politiques de naturalisation plus ouvertes.

Alors que les Turcs, les Serbes, les Croates et les ressortissants de nombreux autres États doivent abandonner leur nationalité d'origine avant de pouvoir acquérir la nationalité allemande, deux groupes sont exempts de cette règle : d'abord les ressortissants de tous les États membres de l'Union européenne et de la Suisse, ensuite les ressortissants d'États qui, comme la Grèce ou le Maroc, refusent toute dénaturalisation, ou qui y opposent des conditions inacceptables, comme l'Iran. Suite à une décision commune des ministères des Affaires Intérieures et Extérieures, les Israéliens aussi sont privilégiés. ${ }^{(13)}$ De fait, cette situation juridique crée un système qui relègue les ressortissants de certains États à des positions désavantagées. Les ressortissants turcs, et d'autres avec eux, sont défavorisés, alors que pour eux la double nationalité serait particulièrement importante. Par ailleurs, étant donné le dynamisme économique de la Turquie, il est dans l'intérêt bien compris de l'Allemagne de favoriser l'émergence de cadres qui soient chez eux dans les deux pays. En revanche, les citoyens d'États membres de l'Union européenne ne voient pas l'intérêt d'acquérir la nationalité allemande, parce qu'à l'exception du droit de vote aux niveaux régional et fédéral ils jouissent presque exactement des mêmes droits que les Allemands.

\section{Des taux de naturalisation inégaux selon les régions et selon les communes}

Puisque le droit est le même dans toute la République fédérale, et puisque selon l'article 3 de la Loi fondamentale toutes les personnes sont égales devant la Loi, on ne peut qu'être surpris de constater des taux de naturalisation fort différents selon les Länder et selon les communes. Le tableau 4 page 75 montre que le Land le plus ouvert en la matière affiche un taux de naturalisation deux fois supérieur à celui du Land le plus fermé. Cela vaut de la même manière pour les anciens que pour les nouveaux Länder dans ces derniers, les taux sont plus bas parce que la durée d'installation des étrangers y est inférieure.

Au niveau communal, on trouvera des différences encore plus importantes qui s'ajoutent à celles entre les Länder. Ainsi, en 2005, le Landkreis ${ }^{(14)}$ Mayen-Koblenz 
Tableaux 4 : taux de naturalisation des Länder allemands en 2006.

Source : Office statistique fédéral, Fachserie 1, Reihe 2.1, Bevölkerung und Erwerbstätigkeit. Einbürgerungen 2006, Wies-baden, 2007, 15. Graphique de l'auteur.

était le plus ouvert de tous les Landkreis et villes de Rhénanie-Palatinat, avec un taux ce de 2,99\%, loin devant le Landkreis Kusel qui affichait un taux de 0,57 \%. On notera qu'en Rhénanie-Palatinat, les Landkreis avec les nombres d'étrangers les plus bas affichent aussi les taux de naturalisation les plus bas, alors que l'effort administratif aurait dû y être moindre. Certains Landkreis se distinguent par des lourdeurs administratives telles que même les naturalisations de droit, et plus encore les procédures où les autorités disposent d'une marge d'interprétation, ont très peu de chances d'aboutir. Une enquête, datant de 2001, montre qu'en France il existe des différences tout aussi importantes entre régions et départements. ${ }^{(15)}$

\section{L'application de la loi n'est pas la même partout en Allemagne}

Pour ce qui concerne l'Allemagne, ces divergences en matière d'application de la loi peuvent s'expliquer par quatre grands groupes de facteurs.

1. Sur le plan administratif, il faut d'abord compter la durée des procédures et l'or- 
ganisation des administrations ainsi que la quantité et la qualité du personnel chargé de ces dossiers. De nombreux témoignages font état d'administrations débordées qui ralentissent les procédures et découragent les requérants. Or, la durée de la procédure est tout sauf neutre. Si les requérants Alors que la Bavière
persiste à refuser dans
toute la mesure du
possible d'accepter le
maintien de nationalités
antérieures, d'autres
autorités se montrent
plus ouvertes. doivent craindre d'avoir à attendre plusieurs années, laissés en plan entre leur ancienne et leur nouvelle nationalité, les effets dissuasifs sont garantis, en particulier s'il s'agit de sujets jeunes, en pleine phase de construction. En revanche, un traitement rapide, sans vexations, provoque des effets de mimétisme ; une naturalisation réussie peut en entraîner d'autres. Le choix des administrations compétentes en la matière dépend des autorités locales, et les solutions varient en conséquence. Elle vont du bureau de l'état civil jusqu'au registre des résidants, en passant par le service des étrangers, non sans exclure des cas où plusieurs administrations interviennent en parallèle. À la différence de la situation en France, où la procédure d'option pour des jeunes prévoit qu'à défaut d'une décision dans les six mois après le dépôt de la demande, le requérant est naturalisé d'office, la procédure en Allemagne peut s'éterniser indéfiniment.

2. Ensuite, il faut noter que la procédure allemande dépend d'actes juridiques d'autres États, ce qui rend les requérants dépendants et redevables, et ce qui peut dans le pire des cas les exposer à des chantages. Ainsi, pour exclure des nationalités multiples, les autorités tendent à exiger des preuves écrites de la déchéance de l'ancienne ou des anciennes nationalités. Une fois sa requête examinée, l'étranger ne reçoit d'abord qu'une promesse ferme de naturalisation. Ensuite, il doit s'adresser à l'État (ou les États) dont il possède la nationalité pour y déposer une requête de dénaturalisation, acquitter les frais afférents et satisfaire à toute autre exigence éventuelle de son ou de ses pays d'origine. C'est donc au moment où l'immigré veut se tourner vers l'Allemagne qu'il se trouve dans la situation paradoxale d'avoir à se soumettre aux exigences des autorités d'autres pays. De plus, les autorités allemandes n'ont aucune prise sur la durée des procédures dans ce ou ces pays, dont on sait par ailleurs qu'elles peuvent se prolonger pendant des mois ou même des années. ${ }^{(16)}$

Cette procédure est à l'opposé de la pratique d'autres États. En France, le requérant se verra notifié par écrit qu'en cas de problème dû à des nationalités multiples, il en est seul responsable, sans pouvoir se retourner vers l'État français. Aux États-Unis, il doit solennellement abjurer tout liens et loyautés envers d'autres 
États. Qu'il le fasse réellement, et comment il le fait, reste entièrement son affaire. En Suède, les étrangers ayant résidé légalement dans le pays pendant cinq ans reçoivent une carte postale les informant qu'ils peuvent maintenant déposer une demande de naturalisation, sans avoir à renoncer à leur ancienne nationalité. Dans des pays comme le Japon ou les Pays-Bas, qui ne reconnaissent plus les nationalités multiples, les requérants doivent s'engager à se démettre de leur ancienne nationalité dans un laps de temps défini ; aux Pays-Bas, celui-ci est de trois ans. Pendant cette période de transition, ils possèdent les deux (ou plusieurs) nationalités et ne courent aucun risque de devenir apatrides.

Selon la situation dans l'État d'origine, la procédure allemande peut générer des frais élevés, provoquer des retardements, des demandes de pots-de-vin, des chantages par des services secrets ou des organes de répression, voire des conséquences négatives pour des parents restés dans le pays d'origine. La procédure n'est pas régie par la loi, mais seulement par le décret d'application du 13 décembre 2000 relatif au droit antérieur de la nationalité, qui n'a pas encore été adapté à la nouvelle situation juridique. Il a été maintes fois démontré que la procédure passant par une promesse de naturalisation, aussi ferme soit-elle, s'avère très souvent impraticable et dissuasive ; elle est un obstacle à l'intégration. Elle oblige des personnes qui veulent être quittes du gouvernement de leur ancien pays à entrer en contact avec celui-ci. Tout compte fait, il vaudrait mieux abandonner cette promesse, et inciter la personne nouvellement naturalisée pour autant et aussi longtemps que cela paraît nécessaire à déposer une déclaration de désistement de l'ancienne nationalité auprès de l'État de la nationalité antérieure.

3. En outre, il est important de diffuser des informations ciblées, qui devraient aussi viser à modifier le climat dans les services concernés ainsi que les attitudes des fonctionnaires. Alors que la Rhénanie du Nord-Westphalie a opté pour une campagne d'information active, la Bavière compte selon son ministère de l'Intérieur sur les "médias et institutions qui utilisent la langue du pays d'origine".

4. Enfin, l'interprétation de la loi au regard des possibilités de garder la ou les nationalités antérieures est une autre source de divergences. À ce sujet, les différences entre les collectivités territoriales sont énormes. Alors que la Bavière persiste à refuser dans toute la mesure du possible d'accepter le maintien de nationalités antérieures, d'autres autorités se montrent plus ouvertes. Ainsi, la ville de Coblence, leader des naturalisations en Rhénanie-Palatinat, a permis aux trois quarts de tous les requérants de garder leur ancienne nationalité. Dans ce Land, ces cas représentaient 46,5 \% en 2005 et 55,0 \% en 2006. Cela dit, même en Bavière, le Land le plus réservé à cet égard, le nombre de détenteurs de plusieurs nationalités ne cesse de monter. 


\section{Notes}

1. Cette contribution est basée sur une brochure du même auteur, publiée par la fondation Friedrich Ebert, sous le titre "Einbürgerung. Rahmenbedingungen, Motive und Perspektiven des Erwerbs der deutschen Staatsangehörigkeit", et disponible sur le site www.fes.de/wiso/

2. Terme intraduisible qui, dans le droit constitutionnel allemand contemporain, ne désigne rien d'autre que la totalité des citoyens d'un État, en l'occurrence la RFA. (N.d.T.)

3. BVerfGE vom 21/5/1974, 239.

4. Bundestags-Drucksache $11 / 1990,48$.

5. Böcker, Anita, Michalowski, Ines, Thränhardt, Dietrich, "Zum Vergleich mit den Niederlanden" ("Succès et échecs des politiques d'intégration. Réévaluer les modèles allemand et néerlandais”), in : Pélissier, Michel, Paecht, Arthur (Hg.), Les Modèles d'intégration en question. Enjeux et perspectives, Paris : Institut de Relations Internationales, Presses Universitaires de France, 2004, p. 25-46.

6. Betriebsräte : conseils d'entreprise. À la différence des comités d'entreprise français, ces conseils, auto-organisés, sont exclusivement composés de salariés et représentent les intérêts de ces derniers vis-à-vis de l'employeur. (N.d.T.)

7. Industriegewerkschaft Metall : syndicat unique dans l'industrie métallurgique, IG Metall compte aujourd'hui environ 2,4 millions d'adhérents. (N.d.T.)

8. Brubaker, Rogers, Citoyenneté et nationalité en France et en Allemagne, Paris, 2000 ; Castles, Stephen, "Democracy and Multiculturalism in Europe", in : Holmes, Leslie, Murray, Philomena (Hg.), Citizenship and Identity in Europe, Aldershot, 1999, p. 59. 9. Hagedorn, Heike, Wer darf Mitglied werden? Einbürgerung in Deutschland und Frankreich im Vergleich, Opladen, 2001.

10. Modification restrictive, très controversée à l'époque, du droit d'asile, nécessitant une révision de l'art. 16 de la Loi fondamentale (N.d.T.)

11. Des enfants nés en Allemagne de parents étrangers (dont au moins un devait aussi être né en Allemagne) auraient acquis la nationalité allemande si les parents en faisaient la demande avant leur $12^{\mathrm{c}}$ anniversaire ; pour la garder au-delà de leur $18^{\mathrm{c}}$ anniversaire, les enfants auraient dû répudier la nationalité de leurs parents dans l'année suivant ce même anniversaire. (N.d.T.)

12. Gosewinkel, Dieter, Einbürgern und Ausschließen, Göttingen, 2001.

13. Deutschland: Doppelpass bei Israelis erlaubt, Migration und Bevölkerung, 10/2005 ; www.tel-aviv.diplo.de.

14. Collectivité territoriale, plus grande qu'un canton, plus petite qu'un département français. (N.d.T.)

15. Hagedorn, Heike, 2001, op. cit.

16. Lettre du ministère de l'Intérieur du Mecklembourg-Poméranie occidentale à l'auteur, 21 décembre 2007. 